\title{
Ciclo de mejora docente aplicado en Temas de Lingüística General
}

\author{
DiEgo JIMÉnEZ PALMERO \\ Universidad de Sevilla \\ Departamento de Lengua Española, \\ Lingüistica y Teoría de la Literatura \\ djimenez@us.es \\ D.O.I.: http://dx.doi.org/10.12795/JDU.2018.i01.18 \\ Pp.: 321-335
}

\section{Resumen}

Esta comunicación narra la experiencia de la aplicación de un Ciclo de Mejora Docente con un modelo didáctico basado en la investigación y en la reelaboración de las ideas de los estudiantes, dentro del marco de la asignatura optativa "Temas de Lingüística General" del cuarto curso correspondiente al Grado de Filología Hispánica y al Doble Grado de Hispánica y Clásica, impartido en la Universidad de Sevilla durante el segundo cuatrimestre del ejercicio docente 2017/2018. Con una muestra total de 8 alumnos participantes, hemos obtenido unos resultados muy favorables que nos indican la necesaria implantación de este tipo de innovaciones educativas y animan a seguir indagando en el tema con una muestra mayor que corrobore las positivas evidencias aportadas mostradas en este Ciclo de Mejora, que, a su vez, nos han permitido concluir con la consolidación de unos principios didácticos que se llevarán a cabo en los futuros ejercicios docentes.

Palabras clave: Lingüística, Filología, Docencia universitaria, experimentación docente universitaria, Gamificación. 


\section{Breve descripción del contexto}

La asignatura "Temas de Lingüística General", en la que se ha aplicado este Ciclo de Mejora Docente, se imparte en el Grado de Filología Hispánica y en el Doble Grado de Hispánica y Clásica. Es una asignatura optativa del segundo cuatrimestre del último curso con una carga docente de 6 ECTS que se ha impartido en 2018 por el doctor Juan Pablo Mora y por el autor de esta comunicación, Diego Jiménez Palmero. Esta misma asignatura ya había sido previamente objeto de un Ciclo de Mejora en el marco de la REFID (Mora, 2017).

Hay 50 alumnos matriculados que se dividen en diez equipos de trabajo en los que es obligatorio que haya alumnado extranjero y español. Cada equipo crea su propio proyecto que debe estar basado en un reto para el que deben encontrar una solución innovadora y presentarla a sus compañeros y a la sociedad al final del cuatrimestre. Ellos son los encargados de liderar su propio proceso de aprendizaje con la figura de los docentes como guías y facilitadores. Además, también se ha recibido la visita de expertos, profesores visitantes y profesores del proyecto.

El Ciclo de Mejora solo se aplica a dos equipos de la clase, que son los que se han decantado por la gamificación como tema central. Uno trata la gamificación para mejorar la Educación Secundaria con el título "La solución es gamificar... o no" y el otro la aplica a niños convalecientes de cáncer con el proyecto "Heroes Academy". Se ha escogido estos dos grupos formados por ocho alumnos en total, de los que cuatro son españoles y cuatro Erasmus, ya que el profesor encargado del Ciclo investiga sobre gamificación y podrá guiar a los estudiantes hacia una mayor profundización en el tema. De esta manera, intentamos que el conocimiento transmitido sea de la mayor calidad posible (García Pérez, 2007).

El interés educativo de la gamificación es cada vez más amplio (Cortizo Pérez, Carrero García, Monsalve Piqueras, Velasco Collado, Díaz del Dedo y Pérez Martín, 2011; Foncubierta y Rodríguez, 2014). El concepto, nombrado por Nick 
Pelling, surgió en 2003 en el campo de la informática. Junto a esa rama, también el ámbito empresarial ha sido uno de los que más lo han utilizado (Werbach y Hunter, 2012; Marín y Hierro, 2013).

En el campo educativo, la gamificación presenta importantes ventajas. Bíró (2014) destaca un sistema de evaluación que depende de la comunidad y el refuerzo y la existencia de rutas de aprendizaje diversificadas. Además, las ventajas de la docencia gamificada no solo las percibe el alumno, sino que, como manifiestan Cortizo Pérez et al. (2011), también las disfruta el profesor: primero, porque se fomenta el trabajo de aula y segundo, porque se facilita la labor de premiar y permite un mayor control del aprendizaje.

\section{Diseño del Ciclo de Mejora Docente}

Hemos elegido seguir el modelo basado en la reelaboración de las ideas de los estudiantes ya que es el que más se ajusta a nuestras necesidades y las de nuestros alumnos porque la clase sigue un modelo docente de Aprendizaje Basado en Retos que se basa en el principio de investigación y en el que se sigue una dinámica de búsqueda de soluciones innovadoras basada en la metodología Design Thinking (Brown, 2009; Meinel, Leifer y Plattner, 2010; Mclntosh, 2016). Los estudiantes trabajan en un PIC (Proyecto Inicio de Carrera) que elaboran durante el cuatrimestre siguiendo distintas fases (empatizar, definir, idear, prototipar y evaluar).

Por tanto, aplicamos el siguiente esquema (Figura 1) en el que "Pr" significa problematización; "le" es ideas de los alumnos; "Ac" corresponde a la actividad de contraste y " $\mathrm{C}$ " son las conclusiones.

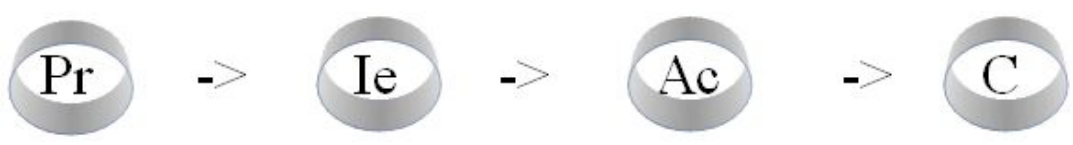

Figura 1. Esquema Modelo basado en la reelaboración de las ideas de los estudiantes. Fuente: De Alba y Porlán (2017, p.41)

Jornadas de Formación e Innovación Docente del Profesorado I № 1 (2018) Esta obra se distribuye con la licencia Creative Commons 
Es decir, buscamos que los alumnos reflexionen sobre lo que investigan en coherencia con el modelo metodológico deseable presentado en De Alba y Porlán (2017). En definitiva, optamos por el planteamiento de un problema y el mantenimiento del mismo a lo largo de todo el proceso de enseñanza, teniendo en cuenta las ideas e intereses de los alumnos y aportando progresivamente nuevas informaciones que contribuyan al tratamiento del problema para finalmente elaborar conclusiones en forma de sintesis final. Por tanto, asumimos pautas metodológicas innovadoras que suponen una novedad con respecto a lo que se acostumbra en las clases de la rama de Humanidades.

Todos estos nuevos conceptos hicieron que en las primeras sesiones los alumnos se encontraran inicialmente algo desorientados. Por tanto, diseñamos un mapa de contenidos y problemas clave que les pudiera servir de guía durante todo el proceso de enseñanza-aprendizaje y que, a continuación, mostramos tal y como se les entregó a los estudiantes que participaban en el Ciclo (Figura 2) para que lo tuvieran como referencia.

Se trata de los aspectos claves del universo que conforma la gamificación. En primera instancia, los dos modelos más seguidos (Chou, 2016; Jiménez, 2015). Le sigue un primer nivel de jerarquía en el que se encuentran la definición clásica de ludificación, la primera tipología de usuarios (Bartle, 1996) y la de recompensas (Iosup y Epema, 2014). En el segundo nivel encontramos lo que no es gamificación y la segunda tipología de usuarios (Marczewski, 2015); y, por último, un tercer nivel con aspectos como las emociones, la narrativa o los avatares. Además, los colores indican el grado de relación entre los contenidos.

También se creó y se acordó un "contrato didáctico" entre docente y estudiantes, que fue firmado al inicio del Ciclo. Siguiendo los principios propuestos por Bain (2012), se realizó buscando compromiso y se consensuó entre todos los agentes educativos participantes. 


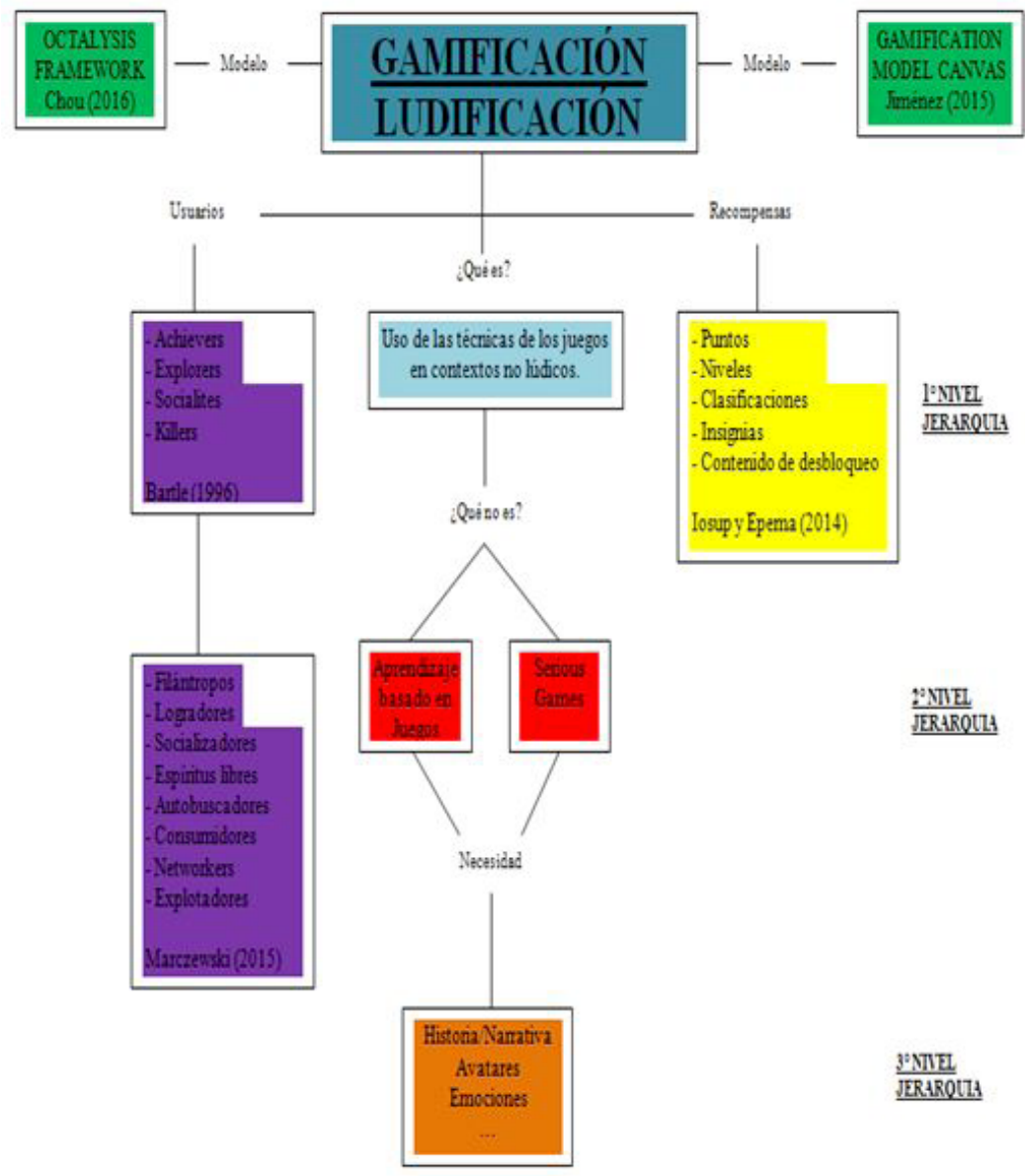

Figura 2. Mapa de contenidos

Además, se diseñó un cuestionario para que los alumnos lo completaran al principio y al final del Ciclo a modo de pretest y postest para seguir la evolución del aprendizaje de los estudiantes. Para crearlo, nos apoyamos en las indicaciones de Rivero y Porlán (2017, p.78) para realizar unas preguntas que obtengan la mayor y mejor información posible: preguntas abiertas y estimulantes, preguntas indirectas, preguntas para interpretar situaciones, preguntas que demanden representaciones gráficas, preguntas comparando fenómenos y preguntas que parten de una negación.

Jornadas de Formación e Innovación Docente del Profesorado I № 1 (2018)
Esta obra se distribuye con la licencia Creative Commons 
Por tanto, aprovechamos esa esencia para formular las siguientes 5 cuestiones que, además, engloban todos los contenidos de este segundo Ciclo de Mejora:

1. Ya que ha elegido usted la gamificación, explique lo que es. [Pregunta abierta, estimulante e indirecta].

2. ¿Le parece una buena gamificación que se utilice el Tabú para fomentar el léxico? [Pregunta para interpretar situaciones].

3. Dibuje las recompensas que considere más adecuadas para los diferentes tipos de usuarios. [Pregunta que demanda representación gráfica].

4. Compare los puntos en común que tienen los siguientes modelos: Octalysis Framework (Chou, 2016) y Gamification Model Canvas (Jiménez, 2015) [Pregunta comparando fenómenos].

5. ¿Por qué no es positiva la gamificación? [Pregunta que parte de una negación].

Las 5 preguntas de este cuestionario pueden representar, de algún modo, una escalera de aprendizaje progresivo, que pretendemos que este Ciclo de Mejora lleve implícito (Figura 3), además de las escaleras que obtenemos con el análisis de los cuestionarios.

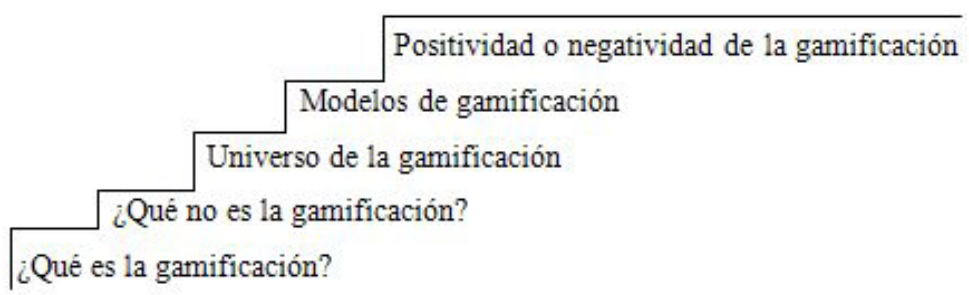

Figura 3. Escalera de aprendizaje progresivo

Jornadas de Formación e Innovación Docente del Profesorado | № 1 (2018) Esta obra se distribuye con la licencia Creative Commons 


\section{Aplicación del Ciclo de Mejora Docente}

\section{Relato resumido de las sesiones}

El desarrollo real del segundo Ciclo de Mejora se ha ajustado casi en su totalidad a lo propuesto inicialmente en el diseño del mismo. La primera sesión fue la toma inicial de contacto recordando lo pactado en el contrato de aprendizaje (30 min.) y realizando el cuestionario pretest (30 min.), junto a la explicación del mapa de contenidos (30 min.).

En la segunda y la tercera sesión se realizó un taller específico para la explicación de los contenidos teóricos (180 min.), que, a su vez, enlazan con una práctica gamificada ya que los estudiantes deben ir consiguiendo superar niveles. Debido a su amplitud se necesitaron dos sesiones que ya estaban contempladas en el diseño.

El profesor, de forma expositiva, explica el universo de la gamificación por niveles que los alumnos deben ir superando con su propia práctica. Se pretende crear conciencia de todo lo que significa y repercute la gamificación ayudando a la creación de sus propios proyectos. Ejemplificamos con un par de las diapositivas del taller (Figura 4).
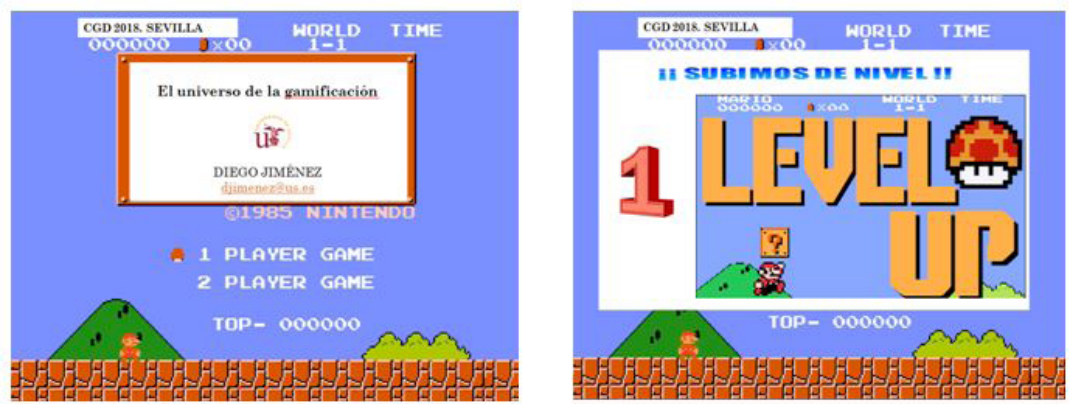

Figura 4. Diapositivas del taller

La cuarta sesión se destinó al modelo basado en la reelaboración de las ideas de los estudiantes (90 min.). En primer lugar, como problematización (Pr) les preguntamos 
las razones por las que han elegido la gamificación para sus proyectos: ¿Por qué habéis elegido para vuestros proyectos la gamificación?

Con una asamblea abierta se exponen los argumentos dentro de la fase de ideas de los alumnos (Ie). Una vez que todos han participado se comienza con la lectura guiada del artículo de Hanus y Fox (2015) que nos servirá como actividad de contraste (Ac).

Hanus y Fox (2015, p.160) en su experimento concluyen que "algunos elementos relacionados con la gamificación pueden ser muy efectivos, pero los educadores deben tener cuidado con el uso de recompensas ya que pueden ser contraproducentes".

Para finalizar, a modo de conclusiones (C) se realiza una puesta en común de las ideas del artículo que nos lleven a una síntesis común en la que sean los alumnos los que comprueben las ventajas y desventajas de la gamificación.

La quinta y última sesión fue para la evaluación (60 min.) y el cuestionario postest (30 min.). Lamentablemente, no hubo tiempo para completar una autoevaluación bidireccional en la que los estudiantes opinaran sobre la función del docente, así que solo se ha podido realizar de forma unidireccional con las sensaciones del docente.

El enfoque de la evaluación que nos planteamos es mediante el uso de las TIC a través del Kahoot, uno de los instrumentos sugeridos, entre otros, por Rivero y Porlán (2017, p.85). De esta manera, se consigue un acercamiento digital hacia los estudiantes, propio de la época en la que impartimos docencia. Además, también se volvió a pasar el cuestionario para poder valorar el momento postest y poder analizar los datos situando ahora a los estudiantes que han participado en relación con la escalera de aprendizaje desarrollada en la primera sesión. 


\section{Evaluación del aprendizaje de los estudiantes}

A continuación, presentamos el análisis de las respuestas a cada una de las preguntas que se hicieron en el cuestionario tanto pretest como postest, pudiendo interpretarse las respuestas, leídas desde la izquierda hacia la derecha, como escalones de una posible progresión. Este aspecto nos permitirá aproximarnos a la comprobación del grado de conocimiento en el que se encontraban nuestros estudiantes y los aprendizajes conseguidos después de la aplicación del Ciclo (Figuras 5, 6, 7, 8 y 9).

1. Ya que ha elegido usted la gamificación, explique lo que es.

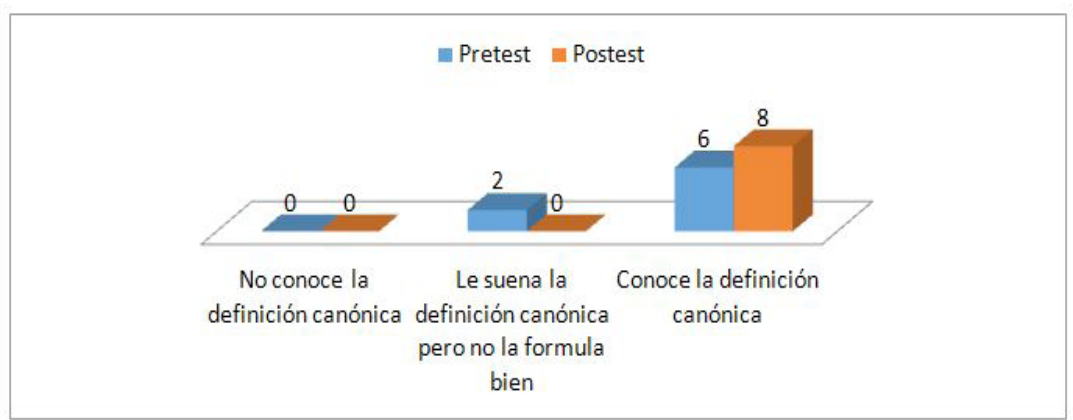

Figura 5. Resultados del cuestionario, pregunta 1.

Conclusión parcial: En el postest dos alumnos suben de escalón mientras que los seis restantes se mantienen en el más alto.

2. Le parece una buena gamificación que se utilice el Tabú para fomentar el léxico?

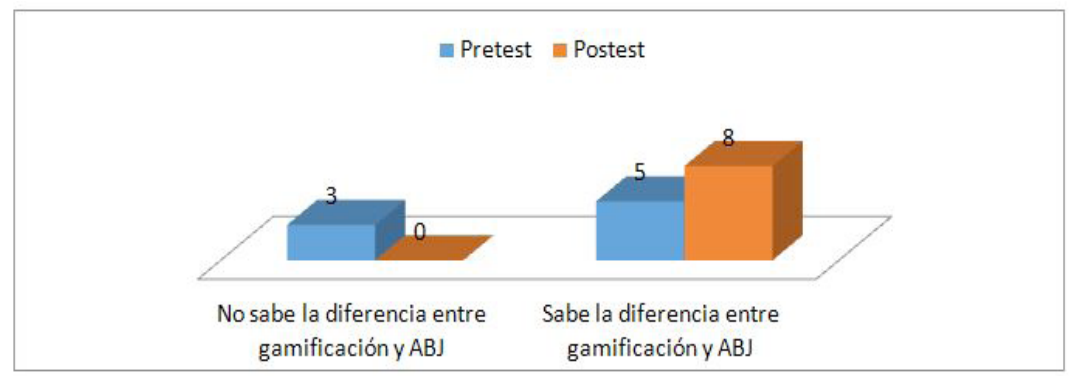

Figura 6. Resultados del cuestionario, pregunta 2.

Jornadas de Formación e Innovación Docente del Profesorado | № 1 (2018) Esta obra se distribuye con la licencia Creative Commons 
Conclusión parcial: Tres alumnos suben de escalón mientras que los cinco restantes se mantienen en el más alto.

3. Dibuje las recompensas que considere más adecuadas para los diferentes tipos de usuarios.

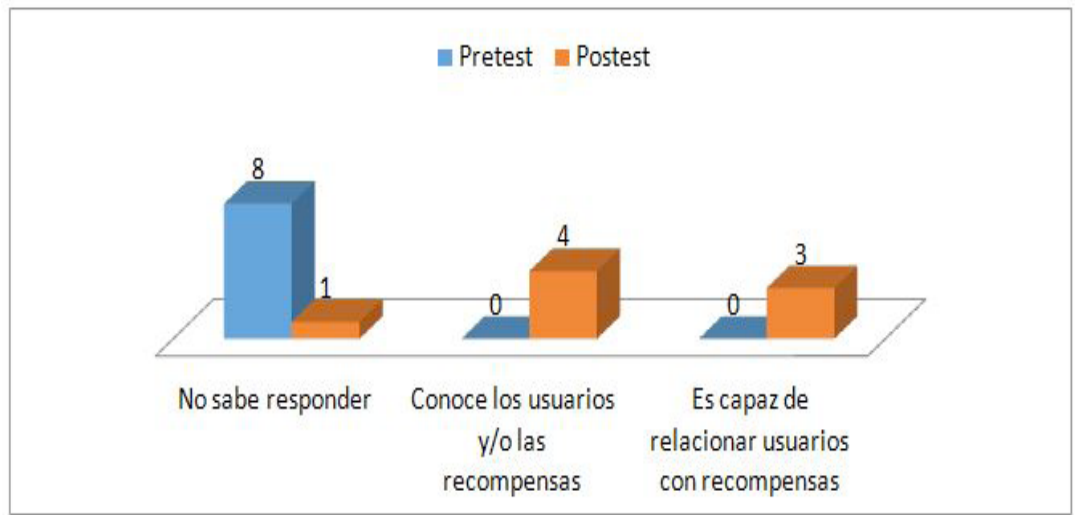

Figura 7. Resultados del cuestionario, pregunta 3.

Conclusión parcial: Un alumno (Erasmus) queda en el mismo nivel mientras que el resto sube uno o dos escalones. Cuatro consiguen alcanzar uno y tres ascienden dos.

4. Compare los puntos en común que tienen los siguientes modelos: Octalysis Framework (Chou, 2016) y Gamification Model Canvas (Jiménez, 2015).

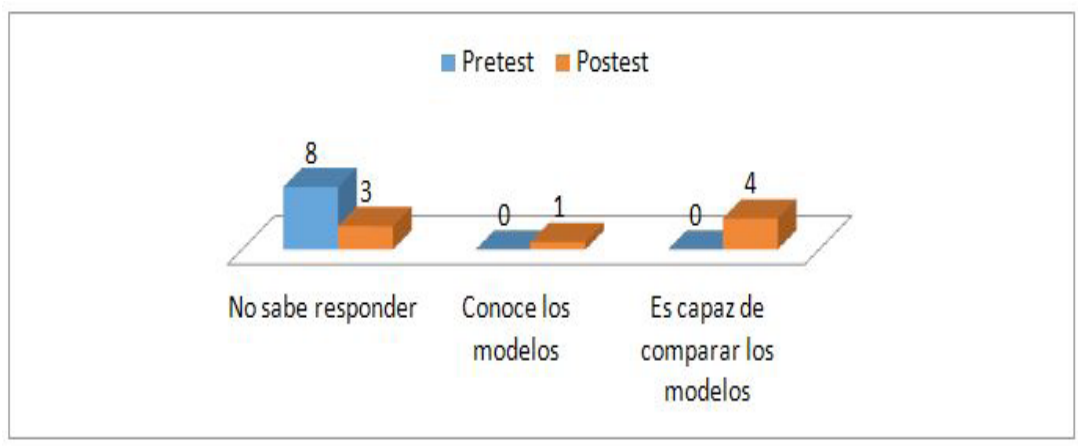

Figura 8. Resultados del cuestionario, pregunta 4.

Jornadas de Formación e Innovación Docente del Profesorado | № 1 (2018) Esta obra se distribuye con la licencia Creative Commons 
Conclusión parcial: Tres alumnos (Erasmus) quedan en el mismo nivel mientras que el resto sube uno o dos escalones. Uno alcanza uno y cuatro suben dos.

5. ¿Por qué no es positiva la gamificación?

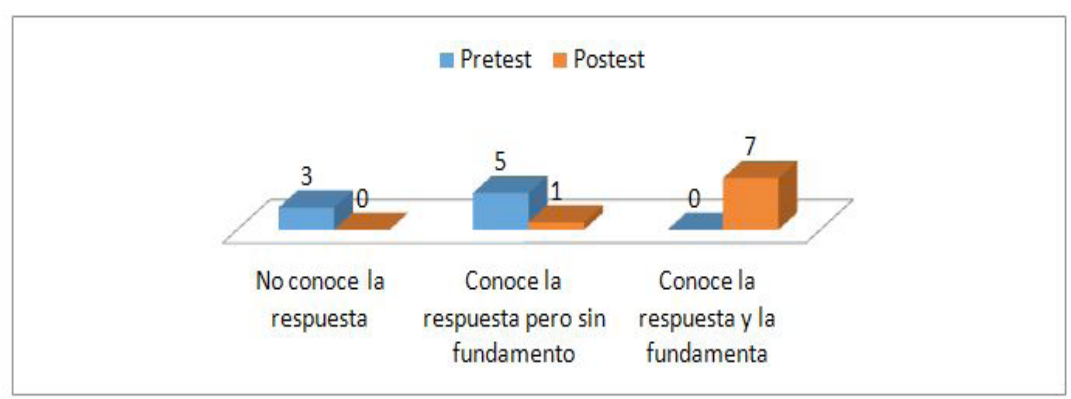

Figura 9. Resultados del cuestionario, pregunta 5.

Conclusión parcial: Todos consiguen mejorar en escalones. Solo uno se queda en el nivel intermedio mientras que el resto logra el máximo.

A modo de conclusión final, podemos señalar que nuestros 8 alumnos partían en el pretest con algunos conocimientos sobre gamificación con un nivel muy inicial que se demuestra observando que contestan mejor las primeras preguntas que son más sencillas. Son lógicos y previsibles esos datos obtenidos ya que son conceptos nuevos para ellos. En el postest observamos cómo los alumnos han mejorado subiendo peldaños de la escalera de aprendizaje correspondiente a cada pregunta. En todas hemos notado una mejoría aunque es cierto que los alumnos Erasmus han sido los que menor progreso han obtenido, ascendiendo solo un escalón o, en ocasiones, quedándose en el mismo. Quizá se deba al factor idioma ya que el ciclo se ha impartido, en todo momento, en español.

De cualquier manera, los resultados obtenidos evidencian que la aplicación de ciclos de Mejora de este tipo en estudiantes universitarios es muy beneficiosa para su mejora del conocimiento. 


\section{Evaluación del Ciclo de Mejora Docente}

El Ciclo de Mejora ha superado con creces las expectativas depositadas en el mismo. Considero que he mejorado como docente siendo más versátil gracias a este curso de innovación. También han sido afectados positivamente mis propios estudiantes ya que han mejorado considerablemente su propio proceso de aprendizaje. Digna de elogio ha sido la predisposición de ellos en todo momento. Probablemente, esto se deba a que el tema elegido les es de gran interés y fueron ellos mismos los que lo seleccionaron en un primer momento.

Esta asignatura ya partía con un modelo didáctico deseable (García Pérez y Porlán, 2017), como es el basado en la investigación. No obstante, hemos podido añadir y fusionar ese marco general con otras referencias pedagógicas como la reelaboración de las ideas de los estudiantes, que es fundamental para fomentar su razonamiento crítico, que es el gran objetivo que me planteo como Principio Didáctico Personal. En definitiva, seguimos lo señalado por Bain (2012) para crear un entorno natural de aprendizaje crítico.

Las programaciones didácticas actuales, tal como se concibe en nuestro sistema educativo, consisten en un proceso de planificación y evaluación sistemática de la actividad de enseñanza-aprendizaje, que otorga respuesta a las clásicas preguntas sobre qué enseñar (objetivos y contenidos), cómo enseñar (metodología y recursos didácticos), cuándo enseñar (secuenciación) y qué, cómo y cuándo evaluar.

Nuestro propósito es mejorar esa definición pensando en los estudiantes ya que el proceso de enseñanza-aprendizaje no se construye de forma unidireccional. En el fondo, el objetivo esencial es el desarrollo integral de la persona, resultando imprescindible la adopción de las actitudes y valores que, a partir del respeto al pluralismo, la libertad, 
la justicia, la igualdad y la responsabilidad, contribuyan a crear una sociedad más desarrollada y justa.

Asumimos de forma consciente y responsable los propósitos planteados para que el alumnado obtenga el máximo desarrollo posible de las capacidades personales. Por tanto, buscaremos la mejora continua en la búsqueda de involucración por parte de los estudiantes para que, de verdad, aprendan.

Además, tendremos presente el decálogo para una educación de calidad elaborado por Cecilia Braslavsky (2004): 1. El foco en la pertinente personal y social; 2. Convicción, estima y autoestima de los involucrados; 3. Fortaleza ética y profesional de los profesores. 4. Capacidad de conducción de los directores e inspectores. 5. Trabajo en equipo, trabajo conjunto. 6. Alianza entre la escuela y los otros agentes educativos. 7. Currículo claro, adecuado y pertinente en cada nivel. 8. Cantidad, calidad y disponibilidad de materiales educativos. 9. Pluralidad y calidad de las didácticas. 10. Unos mínimos de recursos materiales y de incentivos socioeconómicos y culturales.

Por último, cabe subrayar que para obtener los ansiados objetivos debemos seguir un camino que, en ocasiones, será pedregoso. No queremos pecar de optimistas ni tampoco debemos caer en la tentación del profesor pesimista. Más bien debemos seguir unos ideales planteados, buscar los recursos didácticos necesarios y añadir una gran dosis de esfuerzo y trabajo propio y colaborativo con otros compañeros. De esta manera, aunque somos conscientes de la dificultad, es necesario apuntar alto si queremos superar las barreras de todo tipo que nos encontraremos en el desempeño de la profesión. Seguiremos, para ello, con atención las herramientas y manuales (Bain, 2012; Finkel, 2008; Porlán, 2017) a las que hemos tenido acceso en este Ciclo de Mejora ya que serán muy útiles en los futuros años próximos de docencia. 


\section{Referencias biblográficas}

Bain, K. (2012). What the Best College Students Do. Cumberland, RI, USA: Harvard University Press.

Bartle, R. (1996). Hearts, Clubs, Diamonds, Spades: Players Who Suit MUDs. Journal of MUD Research 1, 1. Recuperado de http://mud.co.uk/richard/hcds.htm (26 de mayo de 2018).

Bíró, G.l. (2014). Didactics 2.0: A Pedagogical Analysis Of Gamification Theory From A Comparative Perspective With A Special View To The Components Of Learning. Procedia -Social and Behavioral Sciences. 14, 148-151.

Braslavsky, C. (2014). Diez factores para una educación de calidad para todos en el siglo XXI. Madrid: Fundación Santillana.

Brown, T. (2009). Change by Design: How Design Thinking Transforms Organizations and Inspires Innovation. New York: Harper Collins.

Chou, Y. K. (2016). Actionable Gamification: beyond points, badges and leaderboards. Fremont, CA.: Octalysis Media.

Cortizo Pérez, J. C., Carrero García, F. M., Monsalve Piqueras, B., Velasco Collado, A., Díaz del Dedo, L. I. y Pérez Martín, J. (2011). Gamificación y Docencia: Lo que la Universidad tiene que aprender de los Videojuegos. Actas de las VIII Jornadas internacionales de innovación universitaria. Villaviciosa de Odón (Madrid). Recuperado de http://abacus.universidadeuropea.es/bitstream/ handle/11268/1750/46_Gamificacion.pdf?sequen$\mathrm{ce}=2$ \&isAllowed $=y$ (26 de mayo de 2018).

De Alba, N. y Porlán, R. (2017). La metodología de enseñanza. En R. Porlán (Coord.), Enseñanza universitaria. Cómo mejorarla (pp. 37-54). Madrid: Morata.

Finkel, D. (2008). Dar clase con la boca cerrada. Valencia: Universidad de Valencia.

Foncubierta, J. Ma y Rodríguez, C. (2014): Didáctica de la gamificación en la clase de español. Edinumen, 1-8. Recuperado de https://www.edinumen.es/spanish challenge/gamificacion_didactica.pdf (26 de mayo de 2018).

García Pérez, F.F. (2007). El profesorado y el conocimiento: una relación compleja. Andalucía Educativa, 63, 27-29. Recuperado de http://www. juntadeandalucia.es/educacion/portal/com/bin/ Contenidos/IEFP/ANDALUCIA EDUCATIVA/ANDALUCIA EDUCATIVA/1200924028427 en portada-formacixn del profesorado.pdf (26 de mayo de 2018). 
García Pérez, F. F. y Porlán, R. (2017). Los Principios Didácticos y el Modelo Didáctico Personal. En R. Porlán (Coord.), Enseñanza universitaria. Cómo mejorarla (pp. 93-104). Madrid: Morata.

Hanus, M. D. y Fox, J. (2015). Assessing the effects of gamification in the classroom: A longitudinal study on intrinsic motivation, social comparison, satisfaction, effort, and academic performance. Computers \& Education, 80, 152-161.

Iosup, A. y Epema, D. (2014). An Experience Report on Using Gamification in Technical Higher Education. SIGSE'14, March 3-8, Atlanta GA, USA.

Jiménez, S. (2015). Gamification Model Canvas. Recuperado de http://www.gameonlab.es/canvas/ (26 de mayo de 2018).

Marczewski, A. (2015). Even Ninja Monkeys Like to Play: Gamification, Game Thinking and Motivational Design. San Francisco: Createspace Independent Publishing Platform.

Marín, I. y Hierro, E. (2013). Gamificación: el poder del juego en la gestión empresarial y la conexión con los clientes. Barcelona: Empresa activa.

Meinel, C., Leifer, L. y Plattner, H. (eds.) (2010). Design Thinking: Understand-Improve-Apply. Berlin: Springer.

Mclntosh, E. (2016). Pensamiento de diseño en la escuela: cómo lograr que surjan nuevas ideas y hacerlas realidad. Madrid: Ediciones SM.

Mora, J. P. (2017). Una experiencia de cambio docente en Filología. En R. Porlán (Coord.) Enseñanza universitaria. Cómo mejorarla (pp. 169-178). Madrid: Morata.

Porlán, R. (Coord.) (2017). Enseñanza universitaria. Cómo mejorarla. Madrid: Morata.

Rivero, A. y Porlán, R. (2017). La evaluación en la enseñanza universitaria. En R. Porlán (Coord.) Enseñanza universitaria. Cómo mejorarla (pp. 73-92). Madrid: Morata.

Werbach, K. y Hunter, D. (2012). For the win. How game thinking can revolutionize your business. Philadelphia: Wharton Digital Press. 